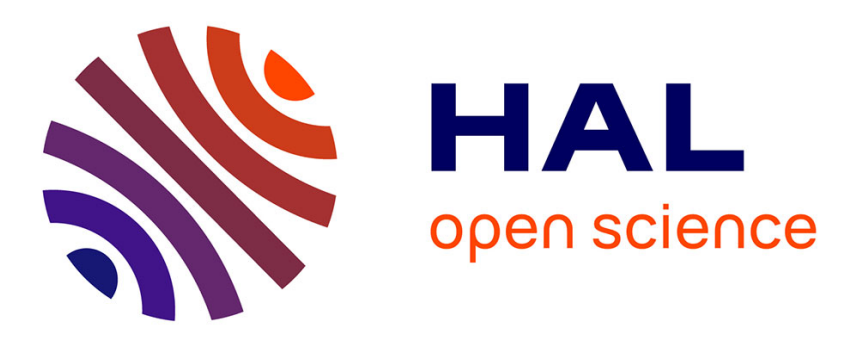

\title{
Interfaces in 100 epitaxial heterostructures of perovskite oxides
}

Jean-Luc Maurice, Dominique Imhoff, Jean-Pierre Contour, Christian Colliex

\section{To cite this version:}

Jean-Luc Maurice, Dominique Imhoff, Jean-Pierre Contour, Christian Colliex. Interfaces in 100 epitaxial heterostructures of perovskite oxides. Philosophical Magazine, 2006, 86 (15), pp.2131-2150. 10.1080/14786430600640460 . hal-00513672

\section{HAL Id: hal-00513672 \\ https://hal.science/hal-00513672}

Submitted on 1 Sep 2010

HAL is a multi-disciplinary open access archive for the deposit and dissemination of scientific research documents, whether they are published or not. The documents may come from teaching and research institutions in France or abroad, or from public or private research centers.
L'archive ouverte pluridisciplinaire HAL, est destinée au dépôt et à la diffusion de documents scientifiques de niveau recherche, publiés ou non, émanant des établissements d'enseignement et de recherche français ou étrangers, des laboratoires publics ou privés. 


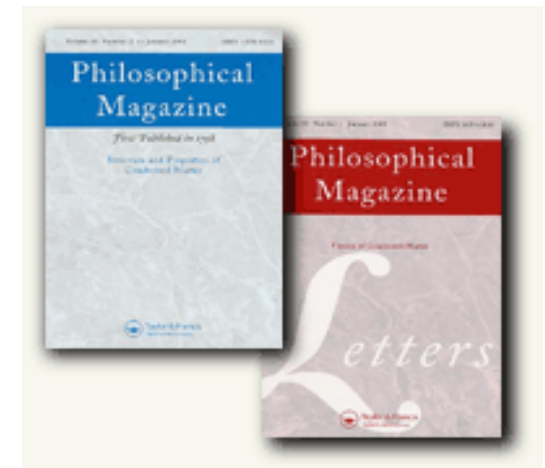

\section{Interfaces in $\{100\}$ epitaxial heterostructures of perovskite oxides}

\begin{tabular}{|c|c|}
\hline Journal: & Philosophical Magazine \& Philosophical Magazine Letters \\
\hline Manuscript ID: & TPHM-05-Apr-0112.R1 \\
\hline Journal Selection: & Philosophical Magazine \\
\hline $\begin{array}{r}\text { Date Submitted by the } \\
\text { Author: }\end{array}$ & $17-O c t-2005$ \\
\hline Complete List of Authors: & $\begin{array}{l}\text { Maurice, Jean-Luc; CNRS/Thales, Unité Mixte de Physique (UMR } \\
\text { CNRS 137) } \\
\text { Imhoff, Dominique; Université Paris Sud, Laboratoire de Physique } \\
\text { des Solides (UMR CNRS 8502) } \\
\text { Contour, Jean-Pierre; CNRS/Thales, Unité Mixte de Physique (UMR } \\
\text { CNRS 137) } \\
\text { Colliex, Christian; Université Paris Sud, Laboratoire de Physique des } \\
\text { Solides (UMR CNRS 8502) }\end{array}$ \\
\hline Keywords: & perovskites, EELS, epitaxy, interfaces, oxide thin films \\
\hline Keywords (user supplied): & \\
\hline
\end{tabular}

\section{$\checkmark$ ScholaroNE"}




\title{
Interfaces in $\{100\}$ epitaxial heterostructures of perovskite oxides
}

\author{
J.-L. MAURICE ${ }^{11}$, D. IMHOFF ${ }^{2}$, J.-P. CONTOUR ${ }^{1}$, C. COLliEX ${ }^{2}$ \\ ${ }^{1}$ Unité Mixte de Physique CNRS ${ }^{2}$ /Thales (UMR CNRS 137), Route départementale 128, \\ 91767 Palaiseau cedex, France, and Université Paris-Sud, 91405 Orsay cedex, France. \\ ${ }^{2}$ Laboratoire de Physique des Solides (UMR CNRS 8502), Bât. 510, Université Paris-Sud, \\ 91405 Orsay cedex, France.
}

\begin{abstract}
The exact perovskite structure has simple cubic symmetry and composition $\mathrm{ABO}_{3}$, where $\mathrm{A}$ is a relatively large cation and $\mathrm{B}$ a smaller one. The choices of A and B cations and the substitutions possible on either site generate a large variety of materials sharing the same base, with relatively small distortions of the size and shape of the cube. As in addition, these oxides often admit oxygen non stoichiometry with or without order to the amount of several per cent, they finally form a vast set of technologically relevant materials: conducting, insulating, ferroelectric, magnetic, superconducting,.... Heteroepitaxy of perovskite oxides allows one to construct atomically sharp interfaces between these materials and therefore to envisage a set of useful heterojunctions. The epitaxy has side effects that may also prove useful: (1) it forces a chemical neighbouring that would not occur naturally, creating a two-dimensional third material, and (2) it imposes a lateral strain. Both of these effects allow one to explore novel, sometimes unforeseen, properties with a strong two-dimensional character. This paper first reviews some of the knowledge that has been accumulated on $\{100\}$ surfaces and interfaces of perovskites, with an emphasis on properties that could be used in future all-oxide microelectronics. It then exposes the case of the interface between the half-metal $\mathrm{La}_{2 / 3} \mathrm{Sr}_{1 / 3} \mathrm{MnO}_{3}$ and the insulator $\mathrm{SrTiO}_{3}$, which plays a key role in the magnetoresistance of magnetic tunnel junctions. It particularly presents thorough electron energy loss spectroscopy measurements that uncover the atomic scale structural and electronic properties of these objects.
\end{abstract}

\footnotetext{
${ }^{1}$ Email : jean-luc.maurice@thalesgroup.com

${ }^{2}$ Centre national de la recherche scientifique
} 


\section{$\S 1$. INTRODUCTION}

Silicon metal oxide semiconductor (MOS) technology has to face in the forthcoming years what is probably the biggest challenge it has ever met with since Moore predicted its fate in 1965 [1]. Due to the scaling down of circuits, silicon's natural oxide $\mathrm{SiO}_{2}$ will no longer fulfil the role of gate dielectric for transistors: if the trend of Moore's law is maintained, this oxide will shortly reach a thickness where electron tunnel trespassing becomes significant. This situation creates a unique opportunity for other candidate materials to replace not only the oxide, but also the whole technology and the material it is based on. Perovskite epitaxial heterostructures, which exhibit a wide variety of useful physical properties, are among these.

The perovskite family includes insulators with a high dielectric constant (e.g. $\left.\mathrm{SrTiO}_{3}\right)$ that can be candidates for use as new gate dielectrics [2, 3]. However, perovskite oxides span a vast range of physical properties: from insulating to semiconducting, conducting and superconducting, or in other respects from ferroelectric or piezoelectric to ferromagnetic, etc., depending on their exact composition. The replacement in standard MOS design of silicon itself by a semiconducting perovskite is under investigation [4]. This multiplicity is obtained within a same simple crystal structure never far from simple cubic. To describe the versatility of the family, Smyth [5] has introduced the concept of 'Perovskite space'. Thanks to the small structural changes from one compound to the other, they can form atomically sharp interfaces in epitaxy. New devices can thus be conceived based on their use in both passive and active parts [6-8].

Of course, as active regions will always include interfaces, one will want the latter as perfect as possible. However 'perfect' it may be, an interface at an atomic scale is an assembly of atoms for which several of the nearest neighbours are 'wrong', so that the physical properties there cannot be those of either of the two crystals in contact. This interface specificity is particularly important in heterostructures where a given crystal site can hold atoms of different valencies, as in the case of diamond and sphalerite semiconductors $[9,10]$ or indeed perovskites. This gives rise to electrostatic frustrations - in insulating or semiconducting systems - or more generally to an interface-specific density of states that may widely differ from that of either of the two crystals in contact, as if the interface were a third material [11]. In other respects, electrostatic frustration at interfaces is also well known at grain boundaries of semiconductors [12] or insulators. The interest for this problem 
in heteroepitaxial perovskite structures is more recent, owing to the more recent development of the atomic-level control of growth for these materials.

The second point that makes heteroepitaxy of perovskites an interesting fundamental study is the interplay in these materials between the distortions and the electronic structure, through the Jahn-Teller effect $[13,14]$. The epitaxial strain must also be considered as a useful tuning parameter for the electronic, magnetic or ferroelectric properties [15].

The aim of the present paper is to shed some light on these yet little investigated interfacespecific properties. As an example, we present the $\mathrm{La}_{2 / 3} \mathrm{Sr}_{1 / 3} \mathrm{MnO}_{3} / \mathrm{SrTiO}_{3}$ (001) interface. $\mathrm{La}_{2 / 3} \mathrm{Sr}_{1 / 3} \mathrm{MnO}_{3}$ (LSMO) is a ferromagnetic metal where only spin up electrons are present at the Fermi level ('half metal') [16-18] and $\mathrm{SrTiO}_{3}$ (STO) is an insulator. Magnetic tunnel junctions made of the stacking LSMO/STO/LSMO, where the LSMO layers are 20-nm thick and the tunnel STO barrier is $\sim 2-\mathrm{nm}$ thick, have exhibited tunnel magnetoresistance (TMR) ratios of about $2000 \%$ [18]. This magnetoresistance decreases with temperature much faster than the magnetisation of the two LSMO films. This has been attributed to the interfaces. After an atomic-scale analysis using electron energy loss spectroscopy (EELS), we propose here to explain how.

In section two, we give general information on the perovskite structure, its defects and the relationships between structure and properties; in section three, we describe how one can grow a perovskite layer epitaxialy on another perovskite and we introduce the importance of the choice of the terminating atomic layer on which to start the growth. In section four, we present our example of interface-specific properties in perovskites, which is that of the LSMO/STO (001) interface. We conclude in section five.

\section{§2. GENERAL INFORMATION ON PEROVSKITE OXIDES}

\subsection{Cubic structure}

The perovskite structure has general formula $\mathrm{ABX}_{3}$, where $\mathrm{A}$ is a relatively large cation, $\mathrm{B}$ a smaller cation, and $\mathrm{X}$ an anion, oxygen in the type compound $\mathrm{CaTiO}_{3}$ and in this paper. Geometrically, the base perovskite unit cell is a cube which can be described in two ways: either with $\mathrm{A}$ ions at the corners and oxygen ions at the centre of the faces forming an octahedral cage around the $\mathrm{B}$ ion in the middle of the cube, or with $\mathrm{B}$ ions at the corners and the $\mathrm{A}$ ion at the centre 
of the cube (figure 1). The latter has the advantage of exhibiting the skeleton of corner-sharing oxygen octahedra. The space group of this base structure is Pm3m. In $<100>$ directions, it is a stacking of $\{200\}$ planes of alternating compositions $\mathrm{AO}$ and $\mathrm{BO}_{2}$, which indicates that two types of $\{100\}$ surfaces can be found.

\subsection{Intrinsic distortions}

Depending on the size ratio of the ions, the oxygen octahedra are most often distorted, rotated or tilted over a small range; the results of these distortions can still be described by a pseudo cubic shape but adopt an orthorhombic (case of $\mathrm{CaTiO}_{3}$ ) or rhombohedral symmetry (figure 2) [19]. The key parameter that causes these symmetry changes is not directly the ionic charge but the ionic radius [20].

\subsection{Distortions and electronic structure}

Jahn and Teller [21] have shown that distorting a highly symmetric atomic arrangement may minimise equilibrium electron energy. The effect that bears their names is particularly active in perovskites on the oxygen octahedra around B-ions (see e.g. ref. 14). An interesting point is the coupling by Jahn-Teller effect of the electronic structure with distortions due to epitaxial strain, in what would be electronic relaxation of strain. The cases where the centre of the oxygen cage is occupied by a $3 \mathrm{~d}$ transition metal have especially been studied: for example copper in hightemperature superconducting cuprates (see e.g. ref. 13), or manganese in colossal-magnetoresistance manganites [22]. Figure 3 summarises the relationships between the d electron energy levels of a $3 d$ transition metal at site $\mathrm{B}$, and distortion of the octahedron in the $\mathrm{z}$ direction, a symmetry change typical of the epitaxial strain.

\subsection{Ionic charge}

The sum of the valencies of ions A and B must be six, with that of B greater or equal than that of A. One has to keep in mind, however, that the picture of six electrons localised on the oxygen ions and six holes on the cations is an oversimplification; the actual localised charge is fractional [23]. In keeping with the pseudo cubic arrangement, the choices for A and B ions are large, as are the possibilities of substitutions on either site and as is the range of oxygen departure from stoichiometry: this is why perovskite oxides span the vast range of physical properties we have 
mentioned in introduction. A graph of some of these properties as a function of composition and point-defect content has been given by Smyth [5].

\section{§3. HETEROEPITAXY OF PEROVSKITES}

\subsection{Growth technique}

The deposition techniques for perovskites have undergone a tremendous progress during the development of high temperature superconductors. Physical as well as chemical deposition methods were tested. These techniques have been reviewed by Scheel et al. [24] for the case of $\mathrm{YBa}_{2} \mathrm{Cu}_{3} \mathrm{O}_{7 \text { - }}$ $\mathrm{x}$. A trend towards atomic layer epitaxy, which is necessary for the control of interfaces, has manifested itself since the beginning of the 1990s [25]. Although classical molecular beam epitaxy has been utilised [26], atomic layer control can be achieved by an a priori less precise technique which is pulsed laser deposition (PLD) [25, 27-30]. The reader can find general information on most of the available techniques in the review papers assembled in the MRS Bulletin of Sept. 1994.

We use PLD to prepare LSMO/STO/LSMO junctions such as the one presented in section 4. Our growth conditions have been described elsewhere [31]. Two points worth mentioning are (1) that the targets are stoichiometric (so that controlling the surface termination of a growing film is not directly possible) and (2) that, in the particular case of the LSMO/STO/LSMO stack, the oxygen partial pressure is maintained during the whole growth at the relatively high value of $\sim 0.3$ torr $(\sim 40$ $\mathrm{Pa})$.

\subsection{Substrate surface}

In order to obtain eventually an atomically sharp interface, it is necessary to start from a perfectly flat surface. More precisely, with a view to using the specific properties of a given stacking sequence, the $\{100\}$ starting surface must be everywhere of a given type, either $\mathrm{AO}$ or $\mathrm{BO}_{2}$. The unavoidable surface steps associated with miscut must have all the same height of a full unit cell. A surface step of half a unit cell changes the nature of the surface from $\mathrm{AO}$ to $\mathrm{BO}_{2}$ (or vice versa) and is undesirable. Of course in reality, reconstructions occur that move the atomic positions [32, 33]. Here, we only consider the type of sequence that a given surface will provoke, which is given by the chemical composition of the last $\{200\}$ plane.

The chemical type of the surface can be characterised using different geometries of Rutherford scattering, e.g. low energy ion scattering spectroscopy (LEISS) or coaxial-impact- 
collision ion-scattering spectroscopy (CAICISS). (These techniques suppose a given atomic order at the surface, usually that of the bulk.) At a microscopic scale, a given type of surface may be recognised by measuring the friction force of a tip (friction force microscopy, FFM) [34]. However, correlating FFM measurements with macroscopic data is not straightforward, so that it is still necessary to perform one's own calibrations to ascertain whether $\mathrm{AO}$ or $\mathrm{BO}_{2}$ is the toughest. In the case of the STO (001) surface, Tanaka et al., by monitoring the last single layer deposited in their layer-by-layer deposition equipment, have found the $\mathrm{SrO}$ termination harder [28]. Iwahori et al. have obtained the same result by comparing FFM and CAICISS data [35].

The domains of equilibrium (oxygen partial pressure, temperature) of a given termination $\mathrm{AO}$ or $\mathrm{BO}_{2}$ - are usually not known. In the case of $\mathrm{SrTiO}_{3}(001)$, it is generally admitted that an anneal around $900^{\circ} \mathrm{C}$, whether in ultra high vacuum [36] or under oxygen flow [33], produces a pure $\mathrm{TiO}_{2}$ surface. Obtaining this surface can also be done by etching in buffered $\mathrm{NH}_{4} \mathrm{~F}-\mathrm{HF}$ [37]. The problem rests with the obviously less stable SrO-terminated surface. Nakamura et al. have observed by LEISS that the surface of a homoepitaxial STO film, grown by PLD on a substrate treated with the Kawasaki method [37], was SrO terminated in the conditions they used [38]. Other authors have used $\mathrm{SrO}[27,39]$ or $\mathrm{Sr}$ [28] targets to achieve SrO termination of their STO substrates or growing films. A drawback of these techniques, especially in the case of $\mathrm{SrO}$ target, is that one does not control whether the (002) atomic plane below the $\mathrm{SrO}$ surface plane is $\mathrm{TiO}_{2}$ as it should, or if it is another SrO plane as in a Ruddlesden-Popper phase (see next section).

In our example of the LSMO/STO/LSMO tunnel junction detailed in section 4, the termination of the starting LSMO surface was one of the questions to be answered, knowing that we had no control on the substrate surface termination. The growth of LSMO onto $\mathrm{TiO}_{2}$-terminated STO is well documented. Yoshimoto et al. [40] on the one hand, Izumi et al. [41] independently, found by CAICISS that LSMO grown at low pressure on this surface was $\mathrm{MnO}_{2}$-terminated. The more recent work of Kumigashira et al. [42], performed by angularly resolved XPS, confirmed this result and also showed that, on SrO-terminated STO conversely, LSMO was La(Sr)O-terminated. Quite unfortunately, our case of relatively high pressure growth is poorly documented. Yoshimoto et al. mention preliminary experiments where the effect of oxygen pressure would be to stabilise the $\mathrm{La}(\mathrm{Sr}) \mathrm{O}(001)$ surface [40]. 
The experimental investigation of any novel phenomena related to a given sequence at the interface poses the difficult problem of preparing surfaces that are unambiguously either AO or $\mathrm{BO}_{2}$ : this is probably one of the major challenges in these studies.

\subsection{Perovskite defects that can be found at epitaxial interfaces}

Quite generally, performing the growth of single crystal films without defects is a challenge. A first source of defects is the mismatch between the crystal parameters of the film and of the substrate and, as growth is generally performed at high temperature, its differential evolution with temperature.

Perovskite oxides respond very differently to epitaxial strain, depending on their plastic and elastic properties: for example $\mathrm{SrTiO}_{3}$ is ductile at room temperature [43], whereas $\mathrm{La}_{2 / 3} \mathrm{Sr}_{1 / 3} \mathrm{MnO}_{3}$ relaxes by fracture when grown at $850^{\circ} \mathrm{C}$ [44]. In all cases, the solution is to prepare layers thinner than the critical thickness $t_{c}$ (at which a first relaxing defect is introduced, see e.g. ref. 45). The actual critical thickness in standard growth conditions is usually neatly higher than the one that can be calculated at equilibrium, which is of the order of $10 \mathrm{~nm}$ for a misfit of $1 \%$ in the case of STO [45]. The actual critical thickness in the case of the growth of LSMO on STO (001), where the parameter misfit is $\sim 0.8 \%$, is $\sim 150 \mathrm{~nm}[44]$.

It is quite important to note that the strain field of misfit dislocations extends over distances typically of the order of their period, at least several $\mathrm{nm}$. This field has an important effect in such materials where bond length and angle are in close relationships with ionic charges, magnetisation or polarisability. Chu et al. have recently shown that distortions by this field in PZT $\left(\mathrm{Pb}\left(\mathrm{Zr}_{0.52} \mathrm{Ti}_{0.48}\right) \mathrm{O}_{3}\right)$ ferroelectric nanodots had the effect of cancelling the polarisation in the regions considered [46].

In addition to a specific value of the crystal parameter, most perovskites have a specific noncubic symmetry so that a given pseudo cubic orientation corresponds to several orientations of the true rhombohedral or orthorhombic phase, which make as many variants in the film, separated by twin boundaries. However, twinning disorientations are small and twin boundaries moreover adapt them over a significant spatial extension [47], so that their effect close to the interface is probably small compared to the epitaxial strain. The manganite $\mathrm{La}_{2 / 3} \mathrm{Sr}_{1 / 3} \mathrm{MnO}_{3}$ that is the object of section 4 is rhombohedral (figure 2) - and twinned in epitaxial films (see ref. [44] for details). 
Another defect which is specific to perovskites originates in the deposition of 'wrong' ions in the first layer (A instead of B or inversely). It leads to a fault in the perovskite stacking sequence at the interface. It can be either an $\mathrm{A}_{\mathrm{f}} \mathrm{O}$ layer on an $\mathrm{AO}$-terminated surface, or a $\mathrm{B}_{\mathrm{f}} \mathrm{O}_{2}$ layer on a $\mathrm{BO}_{2}$-terminated surface (where the index $\mathrm{f}$ indicates that the ion of type $\mathrm{A}$ or $\mathrm{B}$ belongs to the growing film). We are not aware of occurrences of the latter, while the former exists as stacking fault in bulk perovskites. The additional AO plane is then laterally shifted by $1 / 2<110>$ (where the indices refer to the perovskite cube). Structurally, it is equivalent to the replacement in the perovskite $<100>$ sequence of an AO atomic plane by half a rock-salt unit cell of the compound AO; the new sequence actually forms stable phases when repeated, as described by Ruddlesden and Popper [48, 49]. As mentioned in the previous section, the occurrence of such a fault would not be surprising when depositing the rock-salt compound $\mathrm{AO}$ on an $\mathrm{ABO}_{3}$ perovskite substrate.

\section{§4. THE LSMO/STO/LSMO (001) INTERFACES}

\subsection{Generalities}

Tunnel magnetoresistance (TMR) of a magnetic tunnel junction depends on the polarisation of tunnelling electrons [50]. These particles essentially originate from the very last layer of conductive material, at each interface of the two sides of the barrier. It is therefore of prime importance to know how the interfaces modify the bulk propereties.

In the case of magnetic perovskites such as manganites, magnetism and transport are related to the mixed-valency of $\mathrm{B}$ ions $\left(\mathrm{Mn}^{3+/ 4+}\right.$ in LSMO), which in turn depends on the valencies of the surrounding A ions (2/3 $\mathrm{La}^{3+}$ and $1 / 3 \mathrm{Sr}^{2+}$ in LSMO). Ion B may be directly at the interface (type II in our previous work [51]) or 'protected' by an AO layer of its own material (type I in ref. 51). If the manganite is deposited first, type I corresponds to the configuration of figure $4 \mathrm{a}$, and type II to that of figure $4 \mathrm{~b}$. In a sketchy approach, type I, where B (Mn) ions have bulk environment, will be less detrimental to the magnetic properties of the last layer than type II, where the environment of B ions at the interface is 'wrong' for half of it [52]. We shall see below that the two types actually correspond to opposite charge transfers, which will both modify the materials properties. 


\subsubsection{Electrostatic equilibrium}

In order to introduce the electrostatic side of the problem, let us briefly consider a case that has been the object of several works: that of the (001) interface between the insulating perovskites $\mathrm{La}^{3+} \mathrm{Al}^{3+} \mathrm{O}_{3}$ (LAO) and $\mathrm{Sr}^{2+} \mathrm{Ti}^{4+} \mathrm{O}_{3}[27,39]$. When looking at the stacking in the [001] direction, LAO is an alternating pile-up of $\mathrm{La}^{3+} \mathrm{O}^{2-}$ and $\mathrm{Al}^{3+} \mathrm{O}^{2-}$ 2 planes, which carry respectively an electric charge of + lel and - lel per unit cell area, where lel is the positive elementary charge. Whilst on the other hand, STO in this direction is a stacking of $\mathrm{Sr}^{2+} \mathrm{O}^{2-}$ and $\mathrm{Ti}^{4+} \mathrm{O}^{2-}{ }_{2}$ planes that are both neutral. Because the (002) planes are not electrostatically equivalent in LAO and STO, the (001) interface must carry a 'frustration' charge depending on the exact stacking sequence. To keep the configuration of figure 4, let LAO be the substrate; the two following cases can occur: (type I) the starting surface is $\mathrm{LaO}$-terminated as in figure $4 \mathrm{a}$, or (type II) the starting surface is $\mathrm{AlO}_{2}$-terminated as in figure $4 \mathrm{~b}$. In a strictly ionic view with fixed valencies, the former corresponds to doping the structure with half an electron per unit surface cell, and the latter with half a hole. In order to experimentally study these two interfaces, Ohtomo and Hwang have prepared an STO (001) substrate with either $\mathrm{TiO}_{2}$-termination or SrO-termination, for respectively the interfaces of type I and II, and deposited LAO on it. If they did not obtain a doping effect with type II, they indeed obtained a tremendous (actually greater than $1 / 2$ per unit cell) n-doping with type I interface (sequence: $\mathrm{AlO}_{2} / \mathrm{LaO} / / \mathrm{TiO}_{2} / \mathrm{SrO}$ ), with quite high mobilities at low temperature [39].

Although one of the films is conducting in our LSMO/STO interfaces, it is quite useful to submit them to the same analysis, just by replacing LAO by LSMO. Nominally in LSMO, in the absence of oxygen vacancies, the $\mathrm{Mn}^{3.3+} \mathrm{O}^{2-}$ 2 planes have an average electrostatic charge of $-2 / 3$ lel per unit cell area and the $\mathrm{La}^{3+}{ }_{2 / 3} \mathrm{Sr}^{2+}{ }_{1 / 3} \mathrm{O}^{2-}$ ones have a charge of $+2 / 3$ lel per unit cell. In such a case, the charge at the interface would be +lel/3 per unit cell in the case of type I $\left(\mathrm{MnO}_{2} / \mathrm{La}_{2 / 3} \mathrm{Sr}_{2 / 3} \mathrm{O} / / \mathrm{TiO}_{2} / \mathrm{SrO}\right)$, or -lel/3 in the case of type II $\left(\mathrm{La}_{2 / 3} \mathrm{Sr}_{2 / 3} \mathrm{O} / \mathrm{MnO}_{2} / / \mathrm{SrO} / \mathrm{TiO}_{2}\right)$, and in turn this would inject $1 / 3$ electron per unit cell in the former case, and $1 / 3$ hole in the latter. However as both $\mathrm{Ti}$ and $\mathrm{Mn}$ are mixed-valency ions, the actual charge at the interface would depend on whether these carriers be mobile or trapped by the cations, thus moving their valencies. Now introducing the fact that LSMO precisely carries charges by changing the valency of Mn ions, we can give more precision to the picture. In the interface of type I, the valency of Ti ions at the interface would be shifted towards $3+$, or the released electrons would travel into LSMO and shift 
the valency of $\mathrm{Mn}$ ions over a screening length, on average also towards 3+. In the interface of type II on the contrary, the Mn ions at the interface would see their valency shifted on average towards $4+$.

\subsubsection{Tunnel magnetoresistance}

Tunnel magnetoresistance across a manganite/STO/manganite trilayer was first demonstrated by Sun et al. [16]. Our work originated in electron transport experiments carried out by Viret et al. [17] on devices based on two electrodes of LSMO. The tunnel barrier was a two to three-nm thick insulating layer that could be made of either $\operatorname{PrBa}_{2}(\mathrm{CuGa})_{3} \mathrm{O}_{7}, \mathrm{CeO}_{2}$ or $\mathrm{SrTiO}_{3}$. These structures were grown epitaxially by PLD. The highest magnetoresistance was obtained with STO as barrier material, but a sharp decrease was observed above $150 \mathrm{~K}$ (while the Curie temperature of the LSMO layers was above $350 \mathrm{~K}$ ), which was attributed to oxygen vacancies in the vicinity of the interfaces [17]. Some progress has been made since then, but the electron polarisation still decreases faster at the interfaces than in the bulk with temperature [18, 53].

\subsection{Transmission electron microscopy}

In order to understand the origin of this decrease well below Curie point, we have carried out a set of structural measurements by high resolution transmission electron microscopy (HRTEM) on the one hand, and of chemical and electronic measurements by electron energy loss spectroscopy (EELS) with the subnanometre probe of a scanning transmission electron microscope (STEM) on the other hand. We have first analysed the structure and composition of the very junction measured by Viret et al. [17]. Then, in order to study the residual Mn and La EELS signals collected after the apex of the interfaces into the barrier down to zero intensity, we have prepared the same system in identical conditions, where the STO barrier was $5.5 \mathrm{~nm}$ thick (figure 5).

Most of the results of our microscopic investigations have already been published in papers where the reader can find all the technical details concerning our experimental conditions [47, 51, $52,54]$. In the present paper, these results are revisited, and with the support of quite recent publications [55], some routes can be eliminated as possible explanations for the observed behaviour. 


\subsection{Electron energy loss spectroscopy}

We focus here on EELS core edges. In such signals, the energy lost by incident electrons is transferred to core-level electrons that jump to energy levels in the conduction band above the Fermi level. As core levels are very narrow in energy, EELS core edges give in a first order approximation an image of the density of states immediately above the Fermi level, i.e. the density of empty states in the conduction band. In this study, several different edges were recorded and will be discussed in detail, and we will see how this simple approach has to be accepted or modified as a function of the electron levels involved in the transition.

In order to correlate unambiguously the recorded EELS spectra with their exact location of origin in the sample, the spectrum-imaging mode [56] has been used in a VG STEM microscope. This instrument is equipped with a cold field emission source, delivering a minimum probe size of $0.5 \mathrm{~nm}$, with a digitally controlled scanning unit, which can raster the incident probe over the investigated area (1D or 2D patterns and a position displacement step down to $0.2 \mathrm{~nm}$ ) and a parallel EELS spectrometer including a new generation of CCD detector. For each probe position, EELS spectra are acquired over an energy-loss spectral domain incorporating all edges of interest, within typical recording times from tens to hundreds milliseconds for each core-loss spectrum with sufficiently high signal-to-noise ratio to be sensitive to weak changes of fine structures. These huge data sets are analysed and processed a posteriori, using the software that has been developed in our group for elemental mapping, energy loss deconvolution and multivariate statistical analysis. Selected results extracted from these measurements are presented and discussed below. Figure $6 a$ shows a sequence of spectra corresponding to the transition at the interface between the bottom LSMO layer and the insulating layer. Details of the evolution of the fine structures on the three most important signals $\mathrm{O}-\mathrm{K}, \mathrm{Mn}-\mathrm{L}_{2,3}$ and $\mathrm{Ti}-\mathrm{L}_{2,3}$ are extracted for more specific analysis (figures $6 \mathrm{~b}, \mathrm{c}$ and d).

As a general observation, there is no significant difference in any monitored EELS spectral feature between the top and bottom interfaces. This will be considered in section 4.3.3.

A second result is that, when going from the bulk of the LSMO film to the interface, the chemical shift of the Mn- $\mathrm{L}_{2,3}$ edge is small and towards lower energy losses (see figure 9). When compared to the reference work of Abbate et al. [57], this result means that the $\mathrm{MnO}_{2}$ planes are reduced, or electron-doped, in the vicinity of the interface. In our previous publications $[51,52,54]$, 
we had explored several explanation of this electron doping. We had considerered segregation between the La and Sr cations of LSMO (so that there be a higher La/Sr ratio in the vicinity of the interface) and finally excluded it in regard to our recorded elemental profiles [54]. We had also discussed the presence oxygen vacancies in the vicinity of the interface, as proposed by Viret et al. [17]. We were not able to precisely define the main origin of electron doping.

We can now propose a new interpretation scheme following which the electron doping of LSMO at the interface with STO is essentially due to the fact that the stacking sequence is $\mathrm{La}_{0.7} \mathrm{Sr}_{0.3} \mathrm{O} / / \mathrm{TiO}_{2}$ (type I), for which electrostatic equilibrium imposes the presence of extra electrons at the interface (see section 4.1.1). This result is opposite to the hole-doped (type II) configuration assumed by Yamada et al. [58] in a recent study of the same interface. This discrepancy might be related to different growth conditions. Let us now examine all the above mentioned possibilities versus our detailed EELS measurements.

\subsubsection{La/Sr ratio at the STO/LSMO interface}

Figure 7a shows an EELS map exhibiting the ratio of the La-4d and Sr-4p signals over a square area incorporating the two LSMO films on both sides of the STO tunnel junction. It does not show any significant variations over the analysed area in the bulk of the LSMO films. This is quite obvious when we plot profiles across this region, integrated over a few profiles perpendicular to the junction (see figure $7 \mathrm{~b}$ ). Both $\mathrm{La} / \mathrm{Sr}$ and $\mathrm{Mn}$ curves have the half of their maximum value at the same probe positions and the distance between these half maximum value positions corresponds to $5.5 \mathrm{~nm}$, within an accuracy better than $0.5 \mathrm{~nm}$, i.e. the width of the insulating STO layer deduced from HRTEM (figure 5) or annular dark field images. It clearly indicates that there is no increase of the $\mathrm{La} / \mathrm{Sr}$ ratio in the vicinity of the interface.

\subsubsection{Oxygen concentration at the STO/LSMO interface}

The departures of oxygen concentration from stoichiometry are much more difficult to assess. However, they can be searched for in the evolution of different signals when the beam is scanned across the interfaces. It can be directly on the O-K edge or indirectly in the associated transition metal L lines. Let us consider first the family of four spectra exhibiting the detailed profile of the oxygen K-edge, recorded on both reference materials LSMO and STO and at the apex of the two interfaces (bottom and top along the growth direction of the multilayer) (figure 8a). These last two are in excellent agreement, and they can be simulated as a linear combination of the two 
reference ones, with a weighting factor depending on the exact probe position. Non negative least square fit and multivariate statistical analysis reveal no new interface signal different from the mixing between LSMO and STO.

Muller et al. [55] have studied the evolution of the oxygen $\mathrm{K}$ edge for various oxygen deficient strontium titanate foils $\left(\mathrm{SrTiO}_{3-\delta}\right.$ with $\delta$ varying from 0 to 0.25$)$. The spectra that they have recorded are shown in figure $8 \mathrm{~b}$ for comparison with our spectra. The presence of oxygen vacancies in the STO layer when approaching the interfaces should appear through a broadening of the first line towards higher energy loss values and a vanishing of the second peak around $536 \mathrm{eV}$, which is contrary to the behaviour we observe. This effect constitutes a first hint of the absence of oxygen vacancies at the interfaces. However, one must recognize that it is difficult to extract definitive conclusions when considering only the O-K edge, because it is the dominant element present everywhere and weak variations in signal shape could be obscured by the general change of the O-K ELNES signal when evolving from the STO environment to the LSMO one.

An alternative solution, still in the STO layer, is to look for changes of the Ti L 23 lines when approaching the interface, while this signal drops to zero (no Ti present in the LSMO layers). Two spectra recorded at the top and bottom interfaces are superposed in figure 8c, after proper rescaling, on a spectrum corresponding to the centre of the STO tunnel barrier. No significant change can again be detected, while Muller et al. [55] have shown that the presence of oxygen vacancies should give a significant one: the four $\mathrm{Ti}_{23}$ lines, clearly resolved in vacancy-free $\mathrm{STO}$ and in our measurements, should broaden, mix and shift as the oxygen vacancy level $(\delta)$ increases (figure $8 \mathrm{~d}$ ).

The typical splitting of Ti-2p in four lines correspond to the superposition (figure $6 \mathrm{~d}$ ) of intra-atomic effects giving rise to two major lines corresponding to the excitation of the Ti $2 \mathrm{p}_{1 / 2}\left(\mathrm{~L}_{2}\right)$ and $2 p_{3 / 2}\left(L_{3}\right)$, and of the crystal field effect inducing the extra splitting into $t_{2 g}$ and $e_{g}$ states (see figure 3). The presence of oxygen vacancies should indeed change these shapes for several reasons: shift of the average valency of Ti ions from 4+ to 3+, introduction of extra Jahn-Teller contributions to the splitting of the lines either directly or through the induced crystal structure distortions (figure 3). More generally, electron doping of STO would bring a change characteristic of the presence of $\mathrm{Ti}^{3+}$ in the Ti-2p edge [59]

None of these effects occur here. On the contrary, the stability of the detailed shape of the STO Ti $\mathrm{L}_{2,3}$ signal, from the centre of barrier to the interfaces, is quite remarkable on the spectra 
shown in figure 8c, where the energy resolution has been improved down to $0.4 \mathrm{eV}$ using the deconvolution methods developed by Gloter et al.[60]. The clear conclusion at this stage is thus that the concentration of oxygen vacancies in the whole STO layer, up to both interfaces, is close to zero. This statement is consistent with the high-pressure growth conditions (section 3.1).

Applying the same methodology to the manganese $\mathrm{L}_{2,3}$ signal for tracking the possible presence of oxygen vacancies on the LSMO side of the interfaces is less favourable for several reasons associated with the fact that the $3 \mathrm{~d}$ band is not empty in LSMO. Simple arguments on the nature and distribution of empty d states can no longer be used, because as shown by Kurata and Colliex [61] the white line profiles such as the $\mathrm{Mn} \mathrm{L}_{3}$ one are strongly influenced by intra-atomic correlation effects due to the coupling between the $\mathrm{p}$ hole and the d orbitals. We shall see below, however, that oxygen vacancies are not necessary to shift Mn valency and drop the electron polarisation at the interfaces.

\subsubsection{The Mn-2p signal at the interface}

Let us now focus on the evolution of the $M n-2 p_{3 / 2}\left(L_{3}\right)$ line at the interface. Figure 9 exacerbates the separation into two peaks as the displayed spectra correspond to a second derivative mode. Practically in the bulk of the film, the major peak lies at $642 \mathrm{eV}$ and the secondary peak, which is nearly invisible in the bulk LSMO phase in normal mode, points out about $1.8 \mathrm{eV}$ below. When approaching the interfaces with STO, the peak splitting decreases by $0.2 \mathrm{eV}$ and the secondary peak is sharper and its intensity increases. This is valid for both the top and bottom interfaces as they appear here as in other measurements quite symmetrical and equivalent.

In our previous paper dedicated to EELS [54], we have thoroughly discussed this evolution, notably with reference to the work of Abbate et al. [57]. We can now complete our comparisons. Abbate et al. have recorded reference spectra of $\mathrm{Mn}-2 \mathrm{p}$ in the series $\mathrm{La}_{1-x} \mathrm{Sr}_{x} \mathrm{MnO}_{3}$ using X-ray absorption, with $x$ varying from 0 to 0.9 , i.e. Mn valency varying from $3+$ to almost 4+ [57]. They paid a special attention to the two extreme compositions still compatible with the perovskite structure, namely $\mathrm{La}_{0.1} \mathrm{Sr}_{0.9} \mathrm{MnO}_{3}$ and $\mathrm{LaMnO}_{3}$, for which they fitted the experimental spectra with multiplet calculations. Two peaks are present in these calculated and experimental $\mathrm{Mn}-\mathrm{L}_{3}$ lines, very much like in the present EELS spectra. In the series of spectra they have recorded, as the valency of $\mathrm{Mn}$ ion goes from 3+ to 4+, the $\mathrm{Mn}-\mathrm{L}_{3}$ signal undergoes essentially two monotonic changes: (1) the maximum of the $\mathrm{Mn} \mathrm{L}_{3}$ peak shifts by $1 \mathrm{eV}$ towards higher energies, and (2) the splitting between 
the two peaks also increases from 1.5 to $2.4 \mathrm{eV}$ [57]. As in our case the maximum shifts towards lower energies and the peak splitting decreases, we conclude that the $\mathrm{Mn}^{3+} / \mathrm{Mn}^{4+}$ ratio increases at the interface, or else, that the last $\mathrm{MnO}_{2}$ plane in LSMO is electron donated. The apparent increase of the pre-peak intensity in figure 9 is not associated with an increase of the splitting between the two peaks. In our previous work [54], we had proposed that it could be of essentially structural origin, due to the effect of the epitaxial strain on the distortion of $\mathrm{MnO}_{6}$ octahedra. Actually two effects, the increased $\mathrm{Mn}^{3+} / \mathrm{Mn}^{4+}$ ratio and the topology, should reinforce the Jahn-Teller splitting at the interface. In absence of proof, we maintain our earlier proposal that the origin of the enhancement of the low energy peak could be Jahn-Teller effect.

Regarding now the symmetry of the top and bottom interfaces, it implies that the first LSMO film ends up by an AO plane (viz. $\mathrm{La}_{0.7} \mathrm{Sr}_{0.3} \mathrm{O}$ ), and that the thin STO layer ends up on the contrary with a $\mathrm{BO}_{2}$ plane (viz. $\mathrm{TiO}_{2}$ ). We have attributed this reversal to the fact that in the growth conditions used, these surfaces would be thermodynamically stable in respectively LSMO and STO. This is actually confirmed by the preliminary experiment of Yoshimoto et al. [40], in the case of LSMO, while $\mathrm{TiO}_{2}$ is very likely the most stable in STO (see section 3.2).

\subsection{Discussion}

To summarise, we have shown with a careful analysis of EELS experiments that the Mn ions at the LSMO/STO interface present a modified d-band compared to the bulk of LSMO films. The EELS Mn- $\mathrm{L}_{3}$ line exhibits a negative energy shift and a decreased splitting between its two main features, which we have attributed to an increased ratio of $\mathrm{Mn}^{3+}$ or electron doping. Conversely, we have associated the greater sharpness of the minor peak with the interfacial distortion. The only sequence compatible with the increased ratio of $\mathrm{Mn}^{3+}$ is $\mathrm{MnO}_{2} / \mathrm{La}_{0.7} \mathrm{Sr}_{0.3} \mathrm{O} / / \mathrm{TiO}_{2} / \mathrm{SrO}$ (type I). We attribute the shift towards $3+$ to a transfer of electrons from the $\mathrm{TiO}_{2}$ plane of $\mathrm{STO}$, in contact with the $\mathrm{La}_{0.7} \mathrm{Sr}_{0.3} \mathrm{O}$ plane, towards the $\mathrm{MnO}_{2}$ plane of LSMO.

The LSMO/STO interface has been compared, regarding its ferromagnetism, to interfaces of LSMO with other oxides. Yamada et al.[58] have used the sensitivity to surface magnetism of second harmonic generation to probe the interface magnetism of LSMO/STO, LSMO/LAO, and LSMO/two unit-cell $\mathrm{LMO}\left(\mathrm{LaMnO}_{3}\right) / \mathrm{STO}$. They have found that the interface magnetisation was the lowest for LSMO/STO, with the fastest decrease with temperature. They interpreted their results on the assumption that the LSMO/STO interface was a hole donator in their case. It is important to 
note that both possible types of interface - type I (electron donating like here), or type II (hole donating, like in the work of Yamada et al. [58]) - tend to create layers of properties equivalent to those of $\mathrm{La}_{1-\mathrm{x}} \mathrm{Sr}_{\mathrm{x}} \mathrm{MnO}_{3}$ with $x \neq 0.33$. As $x$ departs from 0.33 towards smaller values like here (electron doping), or towards higher values (hole doping), $\mathrm{La}_{1-\mathrm{x}} \mathrm{Sr}_{\mathrm{x}} \mathrm{MnO}_{3}$ has no longer the ferromagnetic properties of LSMO (see e.g. figure 49 in [22]). With such a view, both our approach and that of Yamada et al. [58] explain the decrease of magnetism at the interface. Such a conclusion is however no surprise when considering that the double exchange between Mn ions, at the basis of magnetism in LSMO, must be frustrated at an interface, be it of type I or II.

On the other hand, Garcia et al. [53] have measured the tunnel magnetoresistance of junctions made of LSMO/STO/LSMO, LSMO/LAO/LSMO, and LSMO/TiO $/ 2 S M O$. In that case again, although the low-temperature TMR was higher for the STO barrier, its decrease as the temperature increased was faster than for the LAO one. These junctions were made by one of us in the conditions used for the samples presented here, so that one can assume the same $\operatorname{La}_{0.7} \operatorname{Sr}_{0.3} \mathrm{O}$ termination of LSMO in all junctions. Thus the LSMO/LAO interface would correspond to the sequence $\mathrm{MnO}_{2} / \mathrm{La}_{0.7} \mathrm{Sr}_{0.3} \mathrm{O} / / \mathrm{AlO}_{2} / \mathrm{LaO}$, for which electrostatic equilibrium would impose extra holes. The latter could therefore be less detrimental to the magnetism of LSMO than the electrons donated by LSMO/STO.

Finally, Ab-initio calculations of the different types of interface configurations would be very helpful to fully understand the physics of these systems.

\section{§5. CONCLUSIONS AND PERSPECTIVES}

The goal of the present paper was to exhibit some of the latest advances in the preparation and characterisation of perovskite epitaxial heterostructures. These materials present a very large set of potentialities. Perovskite heterojunctions have been realised to obtain magnetoresistive devices [16-18], MOS structures [4], or 2-dimensional conduction at the interface between two insulators [39]. The effects of heteroepitaxial interfaces on ferroelectricity or bi-ferroicity of perovskites are starting to be explored [15]. Although the heteroepitaxy of perovskites is now a mature subject as it started in the late 1980s with the high temperature superconductors, the atomic-level control of it is only beginning. It appears as a wide field of research, with potential applications in very different areas of the microelectronics world, from ferroelectric memories to the devices that will replace 
CMOS technology. The greatest challenge at this beginning is for thin-film growers. A $\{100\}$ perovskite surface has two variants: this makes it so interesting, but controlling surface termination means controlling the deposition of half a unit cell. This is, to our mind, where the biggest effort is needed. Then come the means of characterising the heterostructures obtained. We hope to have shown here, that in this regard EELS, performed with a subnanometre probe, is a unique tool that allow one to know altogether the nature, the valency, and somehow the site symmetry of the ions present at the interface.

\section{ACKNOWLEDGEMENTS}

The authors wish to thank M. Bibes (Institut d'Electronique Fondamentale, Orsay, France), A. Barthélémy and A. Fert (UMP CNRS/Thales), for fruitful discussions, A. Gloter (LPS Orsay) for critical reading of the manuscript, and É. Jacquet (UMP CNRS/Thales) for the PLD growth.

\section{REFERENCES}

[1] G. E. Moore, Electronics 38114 (1965).

[2] C. Först, C. R. Ashman, K. Schwarz, P. E. Blöchl, Nature 42753 (2003).

[3] J. Robertson, Euro. Phys. J. Appl. Phys. 28265 (2004).

[4] K. Ueno, I. H. Inoue, T. Yamada, H. Akoh, Y. Tokura, H. Takagi, Appl. Phys. Lett. 843726 (2004).

[5] D. M. Smyth, Cryst. Latt. Def. and Amorph. Mat. 18355 (1989).

[6] J. Zhang, H. Tanaka, T. Kawai, Appl. Phys. Lett. 804378 (2002).

[7] A. G. Schrott, J. A. Misewich, R. Ramesh, V. Nagarajan, Mat. Res. Soc. Symp. Proc. 747 V3,8,1 (2003).

[8] K. Shibuya, T. Ohnishi, M. Kawasaki, H. Koinuma, M. Lippmaa, Mat. Res. Soc. Symp. Proc. 747 V3,2,1 (2003).

[9] G. A. Baraff, J. A. Appelbaum, D. R. Hamann, Phys. Rev. Lett. 38237 (1977).

[10] T. Wang, N. Moll, K. Cho, J. D. Joannopoulos, Phys. Rev. Lett. 823304 (1999).

[11] S. Okamoto, A. J. Millis, Nature 428630 (2004).

[12] J.-L. Maurice, Philos. Mag. A 68951 (1993).

[13] B. Raveau, C. Michel, M. Hervieu, D. Groult, Springer series in materials science 15 (1991).

[14] A. J. Millis, Nature 392147 (1998). 
[15] C. Thiele, K. Dörr, L. Schultz, E. Beyreuther, W.-M. Lin, submitted.

[16] J. Z. Sun, W. J. Gallagher, P. R. Duncombe, L. Krusin-Elbaum, R. A. Altman, A. Gupta, Y. Lu, G. Q. Gong, G. Xiao, Appl. Phys. Lett. 693266 (1996).

[17] M. Viret, M. Drouet, J. Nassar, J.-P. Contour, C. Fermon, A. Fert, Europhys. Lett. 39545 (1997).

[18] M. Bowen, M. Bibes, A. Barthélémy, J.-P. Contour, A. Anane, Y. Lemaître, A. Fert, Appl. Phys. Lett. 82233 (2003).

[19] R. S. Roth, J. Res. Natl. Bur. Std. 5875 (1957).

[20] V. M. Goldschmidt, Skrifter Norske Videnskaps-Akad. Mat. Naturv. K1. 28 (1926).

[21] H. A. Jahn, E. Teller, Proc. R. Soc. A 161220 (1937).

[22] J. M. D. Coey, M. Viret, S. von Molnár, Adv. Phys. 48167 (1999).

[23] M. Coey, Nature 430155 (2004).

[24] H. J. Scheel, M. Berkovski, B. Chabot, J. Cryst. Growth 11519 (1991).

[25] M. Y. Chern, A. Gupta, B. W. Hussey, Appl. Phys. Lett. 603045 (1992).

[26] F. Arrouy, J.-P. Locquet, E. J. Williams, E. Mächler, R. Berger, C. Gerber, C. Monroux, J.-C. Grenier, A. Wattiaux, Phys. Rev. B 547512 (1996).

[27] D.-W. Kim, D.-H. Kim, B.-S. Kang, T. W. Noh, D. R. Lee, K.-B. Lee, Appl. Phys. Lett. 74 2176 (1999).

[28] H. Tanaka, H. Tabata, T. Kawai, Thin Solid Films 3424 (1999).

[29] G. Rijnders, G. Koster, V. Leca, D. H. A. Blank, H. Rogalla, Appl. Surf. Sci. 168223 (2000).

[30] A. Ohtomo, D. A. Muller, J. L. Grazul, H. Y. Hwang, Nature 419378 (2002).

[31] J. P. Contour, C. Sant, D. Ravelosona, B. Fischer, L. Patlagan, Jap. J. Appl. Phys 321134 (1993).

[32] G. Renaud, Surf. Sci. Reports 321 (1998).

[33] N. Erdman, K. R. Poeppelmeier, M. Asta, O. Warschkow, D. E. Ellis, L. D. Marks, Nature 419 55 (2002).

[34] J. Fompeyrine, R. Berger, H. P. Lang, J. Perret, E. Mächler, Ch. Gerber, J.-P. Locquet, Appl. Phys. Lett. 721697 (1998).

[35] K. Iwahori, S. Watanabe, M. Kawai, K. Mizuno, K. Sasaki, M. Yoshimoto, Appl. Phys. Lett. 887099 (2000). 
[36] W. Maus-Friedrichs, M. Friedrichs, A. Gunhold, S. Krischok, V. Kempter, G. Bihlmayer, Surf. Sci. 515499 (2002).

[37] M. Kawasaki, K. Takahashi, T. Maeda, R. Tsuchiya, M. Shinohara, O. Ishiyama, T. Yonesawa, M. $\quad$ Yoshimoto, H., Koinuma, Science 2661540 (1994).

[38] T. Nakamura, H. Inada, M. Iiyama, Appl. Surf. Sci. 130-132 576 (1998).

[39] A. Ohtomo, H. Y. Hwang, Nature 427423 (2004).

[40] M. Yoshimoto, H. Maruta, T. Ohnishi, K. Sasaki, H. Koinuma, Appl. Phys. Lett. 73187 (1998).

[41] M. Izumi, Y. Konishi, T. Nishihara, S. Hayashi, M. Shinohara, M. Kawasaki, Y. Tokura, Appl. Phys. Lett. 732497 (1998).

[42] H. Kumigashira, K. Horiba, H. Ohguchi, K. Ono, M. Oshima, N. Nakagawa, M. Lippmaa, M. Kawasaki, H. Koinuma, Appl. Phys. Lett. 823430 (2003).

[43] P. Gumbsch, S. Taeri-Baghbadrani, D. Brunner, W. Sigle, M. Rühle, Phys. Rev. Lett. 87 085505 (2001).

[44] J.-L. Maurice, F. Pailloux, A. Barthélémy, O. Durand, D. Imhoff, R. Lyonnet, A. Rocher, J.-P. Contour, Philos. Mag. 833201 (2003).

[45] J.-P. Contour, A. Abert, A. Défossez, SPIE 2697339 (1996).

[46] M.-W. Chu, I. Szafraniak, R. Scholz, C. Harnagea, D. Hesse, M. Alexe, U. Gösele, Nature Mater. 387 (2004).

[47] R. Lyonnet, J.-L. Maurice, M. J. Hÿtch, D. Michel, J.-P. Contour, Appl. Surf. Sci. 162-163 245 (2000).

[48] S.N. Ruddlesden, P. Popper, Acta Cryst. 10538 (1957).

[49] S. N. Ruddlesden, P. Popper, Acta Cryst. 1154 (1958).

[50] M. Julière, Phys. Lett. A 54225 (1975).

[51] J.-L. Maurice, F. Pailloux, D. Imhoff, N. Bonnet, L. Samet, A. Barthélémy, J.-P. Contour, C. Colliex, A. Fert, Euro. Phys. J. Appl. Phys. 24215 (2003).

[52] F. Pailloux, D. Imhoff, T. Sikora, A. Barthélémy, J.-L. Maurice, J.-P. Contour, C. Colliex, A. Fert, Phys. Rev. B 66014417 (2002).

[53] V. Garcia, M. Bibes, A. Barthélémy, M. Bowen, E. Jacquet, J.-P. Contour, A. Fert, Phys. Rev. B 69052403 (2004). 
[54] L. Samet, D. Imhoff, J.-L. Maurice, J.-P. Contour, A. Gloter, T. Manoubi, A. Fert, C. Colliex, Euro. Phys. J. B. 34179 (2003).

[55] D. A. Muller, N. Nakagawa, A. Ohtomo, J. L. Grazul, H. Y. Hwang, Nature 430657 (2004).

[56] C. Jeanguillaume, C. Colliex, Ultramicroscopy 28252 (1989).

[57] M. Abbate, F. M. F. de Groot, J. C. Fuggle, A. Fujimori, G. A. Sawatski, M. Takano, Y. Takeda, H. Eisaki, S. Uchida, Phys. Rev. B. 464511 (1992).

[58] H. Yamada, Y. Ogawa, Y. Ishii, H. Sato, M. Kawasaki, H. Akoh, Y. Tokura, Science 305646 (2004).

[59] M. Abbate, F. M. F. de Groot, J. C. Fuggle, A. Fujimori, Y. Tokura, Y. Fujishima, O. Strebel, M. Domke, G. Kaindl, J. van Elp, B. T. Thole, G. A. Sawatzki, M. Sacchi, N. Tsuda, Phys. Rev. B. 445419 (1991).

[60] A. Gloter, A. Douiri, M. Tencé, C. Colliex, Ultramicroscopy 96385 (2003).

[61] H. Kurata, C. Colliex, Phys. Rev. B 482102 (1993). 
25/06/2010 Maurice et al. : Interfaces in perovskites

Figure captions

Figure 1. The perovskite unit cell $\mathrm{ABO}_{3}$, seen with $\mathrm{A}$ ions at the corners (a), or $\mathrm{B}$ ions at the corners (b). The ion sizes are smaller than in reality but have the right ratios. Oxygen ions that form the octahedra are not drawn in (b) for clarity.

Figure 2. The rhombohedral unit cell of the manganite $\mathrm{La}_{2 / 3} \mathrm{Sr}_{1 / 3} \mathrm{MnO}_{3}$ (a) still leads to a pseudo-cubic stacking of the oxygen octahedra (b). The crystallographic directions indicated refer to the pseudo cube (where the 'cubic' angle is $90.26^{\circ}$ ).

Figure 3. Effect of varying the environment of a $3 \mathrm{~d}$ transition metal at site $\mathrm{B}$ on its $3 \mathrm{~d}$ electron states: the crystal field (CF) of the oxygen octahedron performs a first removal of degeneracy. An additional removal of degeneracy may occur due a lowering of symmetry associated with the Jahn-Teller effect (JT), the epitaxial strain, or to the presence of an oxygen vacancy.

Figure 4. The two types of interfaces obtained when growing a perovskite $\mathrm{A}_{\mathrm{f}} \mathrm{B}_{\mathrm{f}} \mathrm{O}_{3}$ on the $\mathrm{AO}$ or $\mathrm{BO}_{2}\{200\}$ surface of a perovskite $\mathrm{ABO}_{3}$. Depending on the valency of the cations, they may have opposite electronic properties (see text).

Figure 5. HRTEM image of the sample analysed in figures 6 to 9.

Figure 6. (a) Variations of electron energy loss fine structure (ELNES) across the LSMO/STO/LSMO stack shown in figure 5, using the spectrum-line technique. The evolutions of the O-1s, Mn-2p and Ti-2p edges across the "bottom" interface (where STO is grown on top of LSMO) is detailed in (b) to (d) respectively, after background subtraction. (The top interface however exhibits very similar features [54].) The spatial distance between spectra is $0.47 \mathrm{~nm}$. An energy deconvolution technique has been used to improve energy resolution [60].

Figure 7. (a) Chemical map of the La to Sr ratio over the LSMO/STO/LSMO junction shown in figure 5. The values were extracted from the La- $4 \mathrm{~d}$ and $\mathrm{Sr}-4 \mathrm{p}$ spectrum images. (b) Profiles averaged parallel to the interfaces of the $\mathrm{La} / \mathrm{Sr}$ atomic ratio (full line) and of the absolute $\mathrm{Mn}$ concentration (full circles). Acquisition time: $10 \mathrm{~ms}$ per spectrum. The fact that the two profiles coincide indicates the absence of any significant cation segregation. 
25/06/2010 Maurice et al. : Interfaces in perovskites

Figure 8. Comparisons of ELNES of O-1s (K-edge) and Ti-2p ( $\mathrm{L}_{2,3}$ edge) in the present case $(\mathrm{a}, \mathrm{c})$ and in recordings performed by Muller et al. [55] in $\mathrm{SrTiO}_{3-\delta}$ samples with varying oxygen vacancy concentration $\delta / 3(b, d)$. (a) Comparison between the O-K signals in the bulk of the STO barrier and LSMO films and at the apex of the interfaces: interface signals are averages of the bulk ones. (b) Comparison of the Ti- $\mathrm{L}_{2,3}$ signal from the centre of the barrier with those at the interfaces, after re-scaling for clarity: there are no significant differences. Note the sharpness of the peaks in (a) and (c) when compared to the broadening that should occur in the presence of oxygen vacancies in (b) and (d) (graphs b and d reprinted by kind permission of David Muller).

Figure 9. Evolution of the ELNES of Mn-2p $\left(\mathrm{L}_{2,3}\right)$ edge across the LSMO/STO/LSMO stack of figure 5, displayed in second derivative mode to exacerbate the fine structure. Spectra recorded when the centre of the electron probe is $0.9 \mathrm{~nm}$ within the barrier (where no $\mathrm{Mn}$ is present) best image interface-specific features. The vertical lines highlight a small amplitude shift towards lower energies when going from LSMO to the interfaces. 


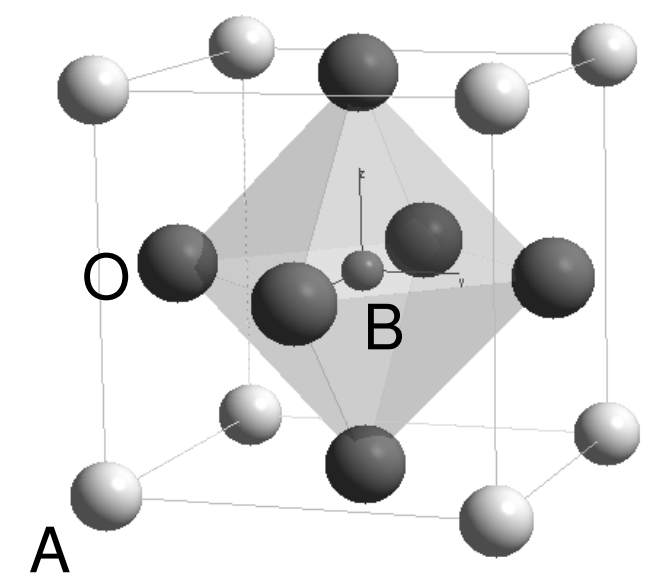

(a)

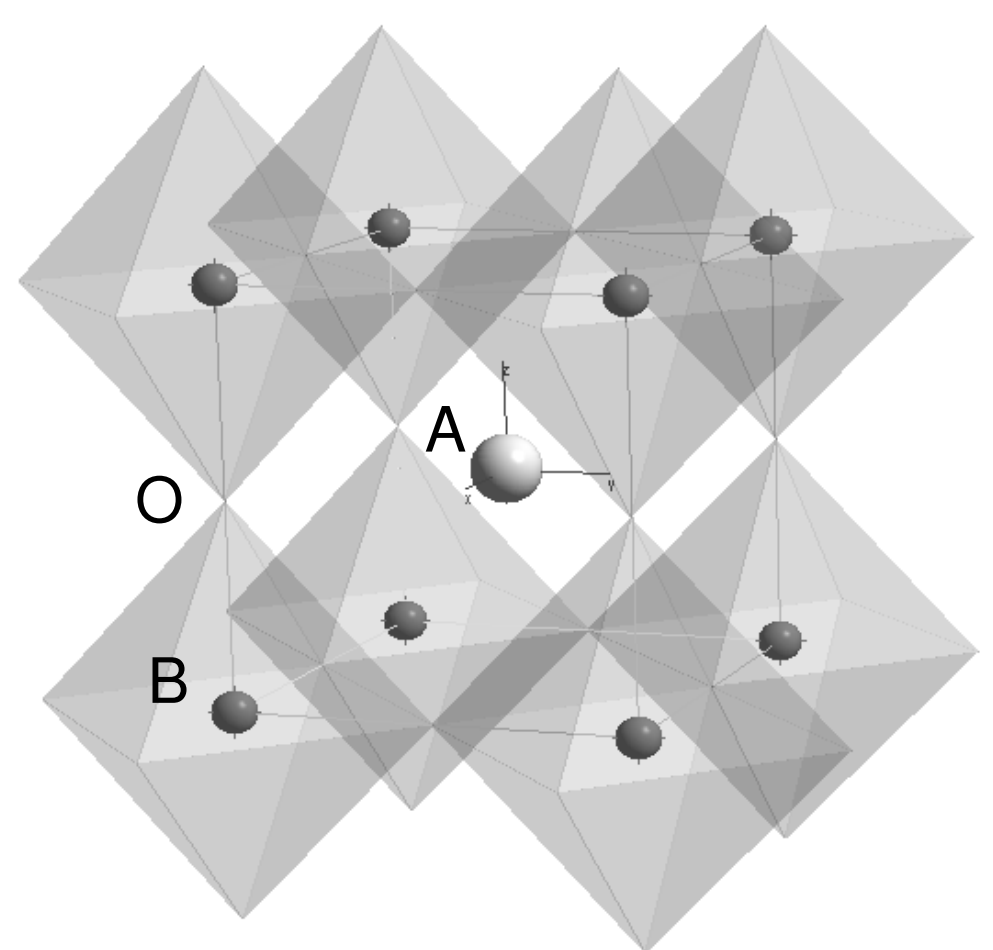

(b)

Fig. 1 


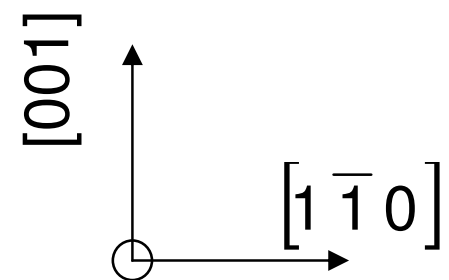

[110]

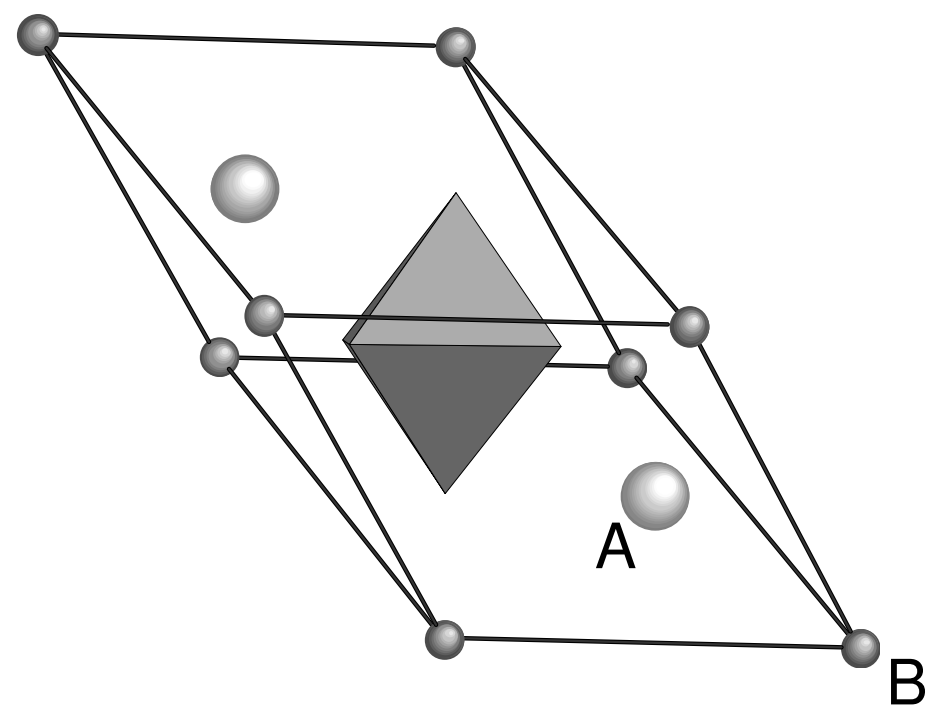

(a)

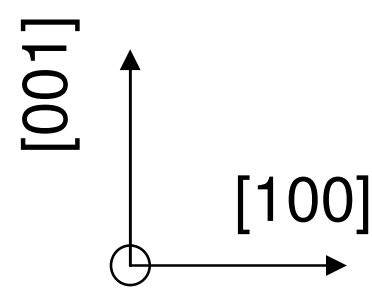

[010]

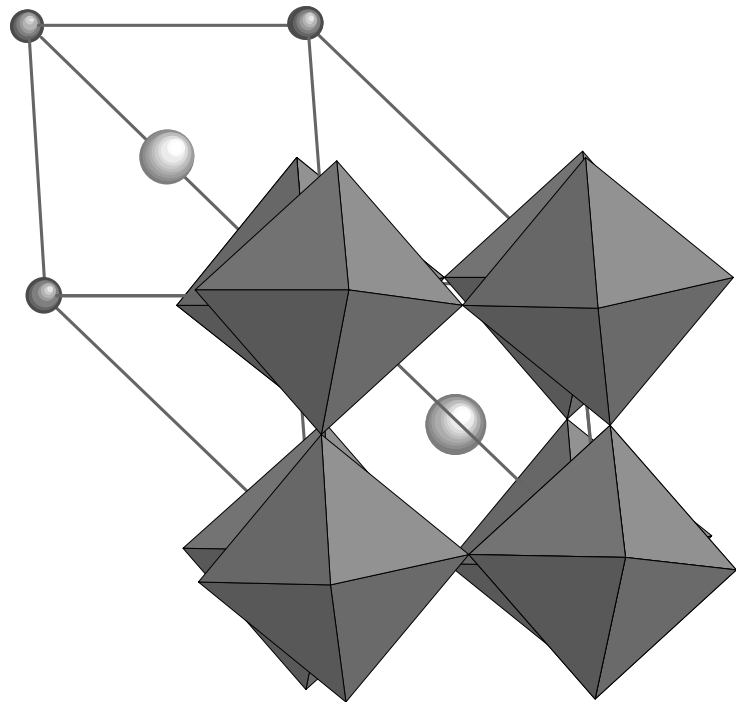

(b)

Fig. 2

Maurice et al.: Interfaces in perovskites 

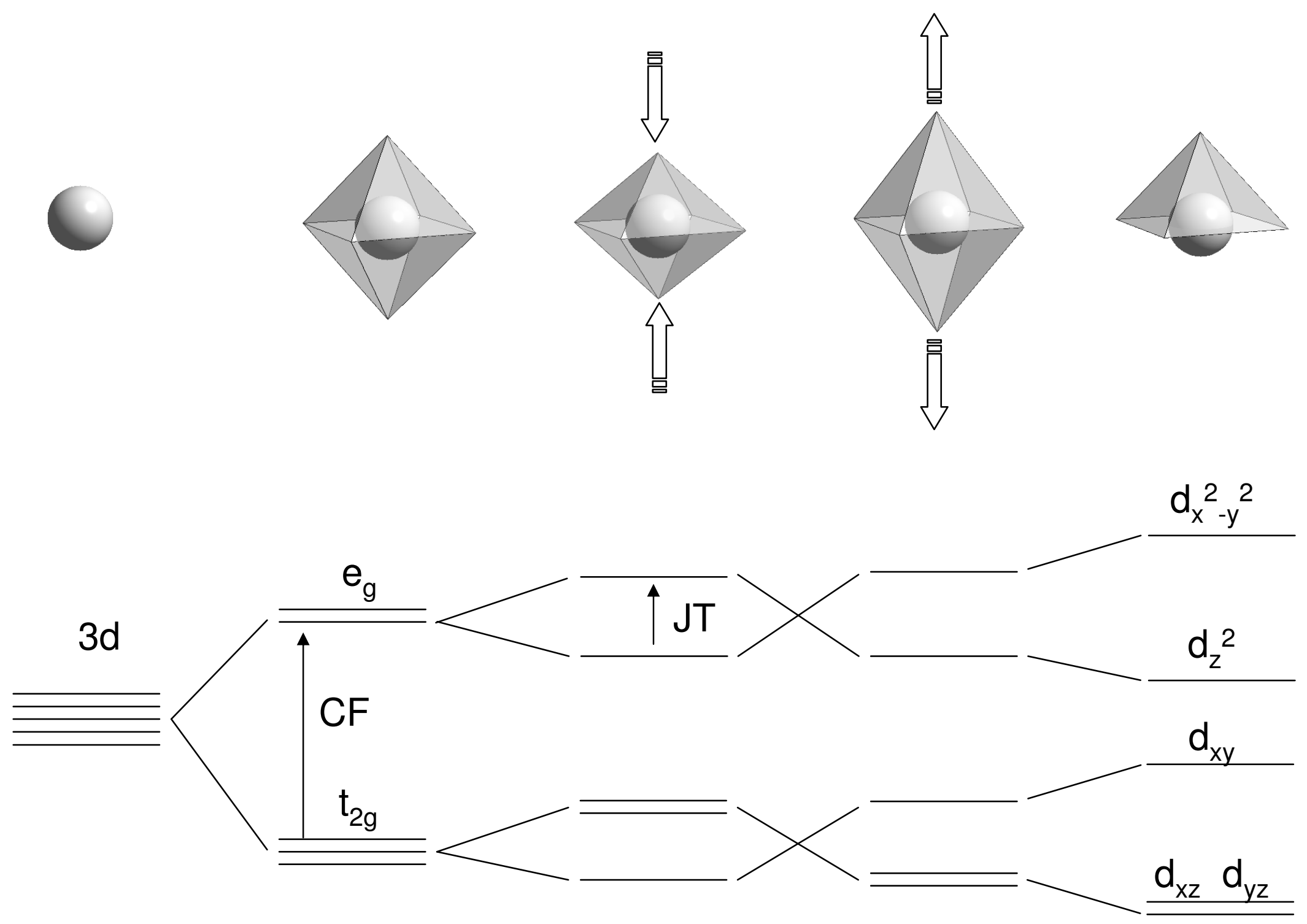

Fig. 3

Maurice et al.: Interfaces in perovskites 


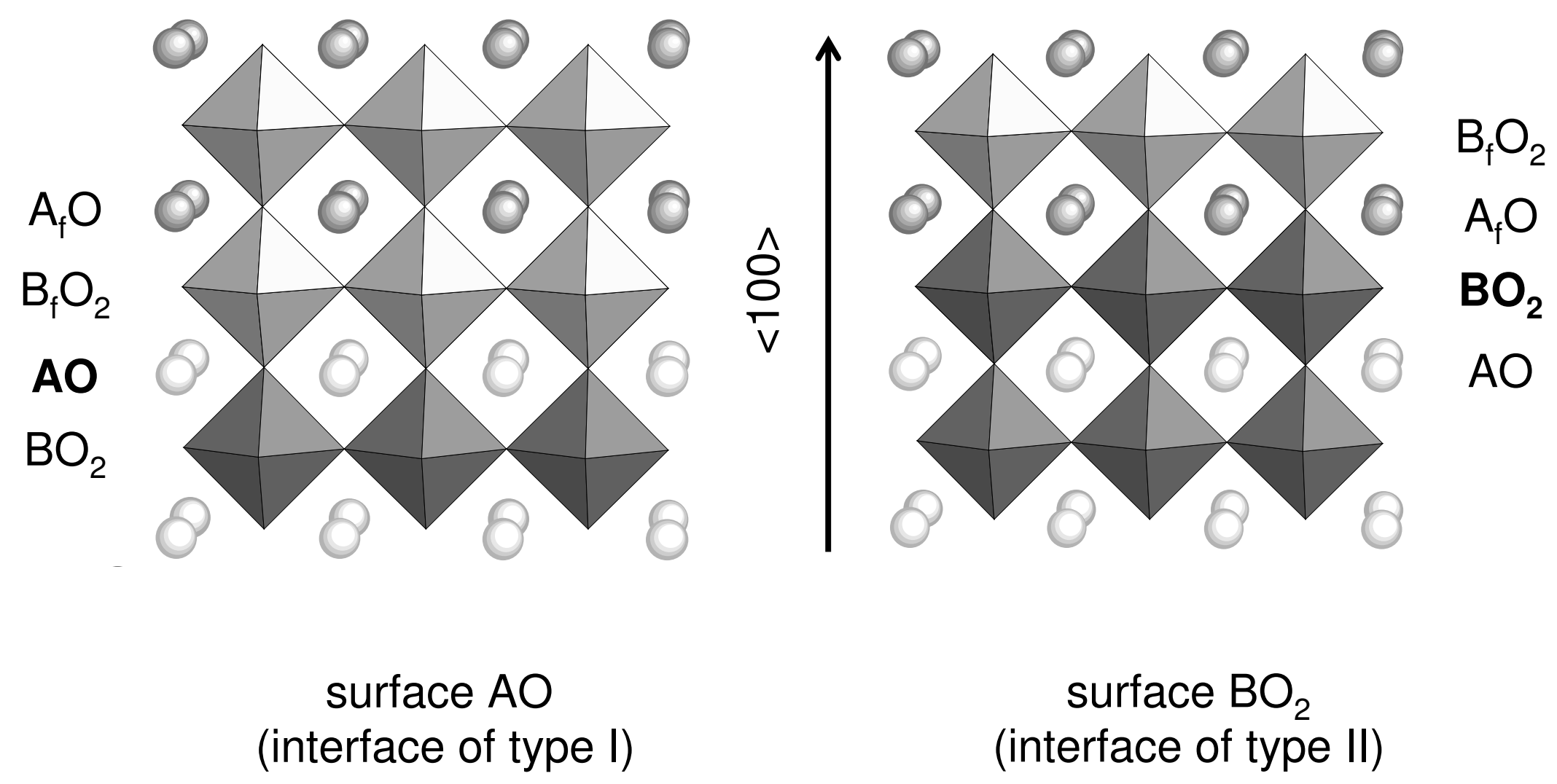

Fig. 4 


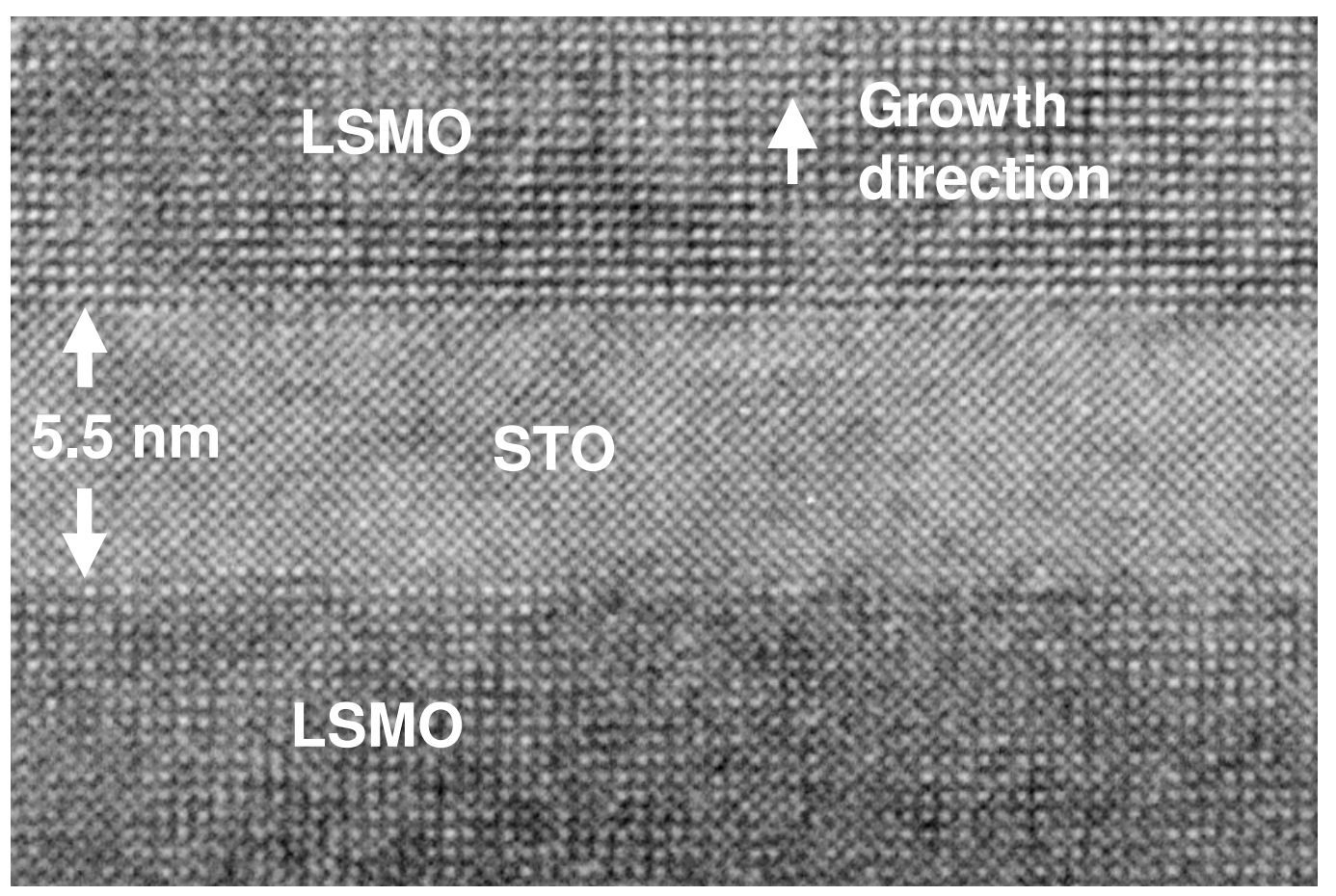

Fig. 5 
a

$3.10^{6} \mathrm{e}^{-} \mathrm{Ti}-2 \mathrm{p}$
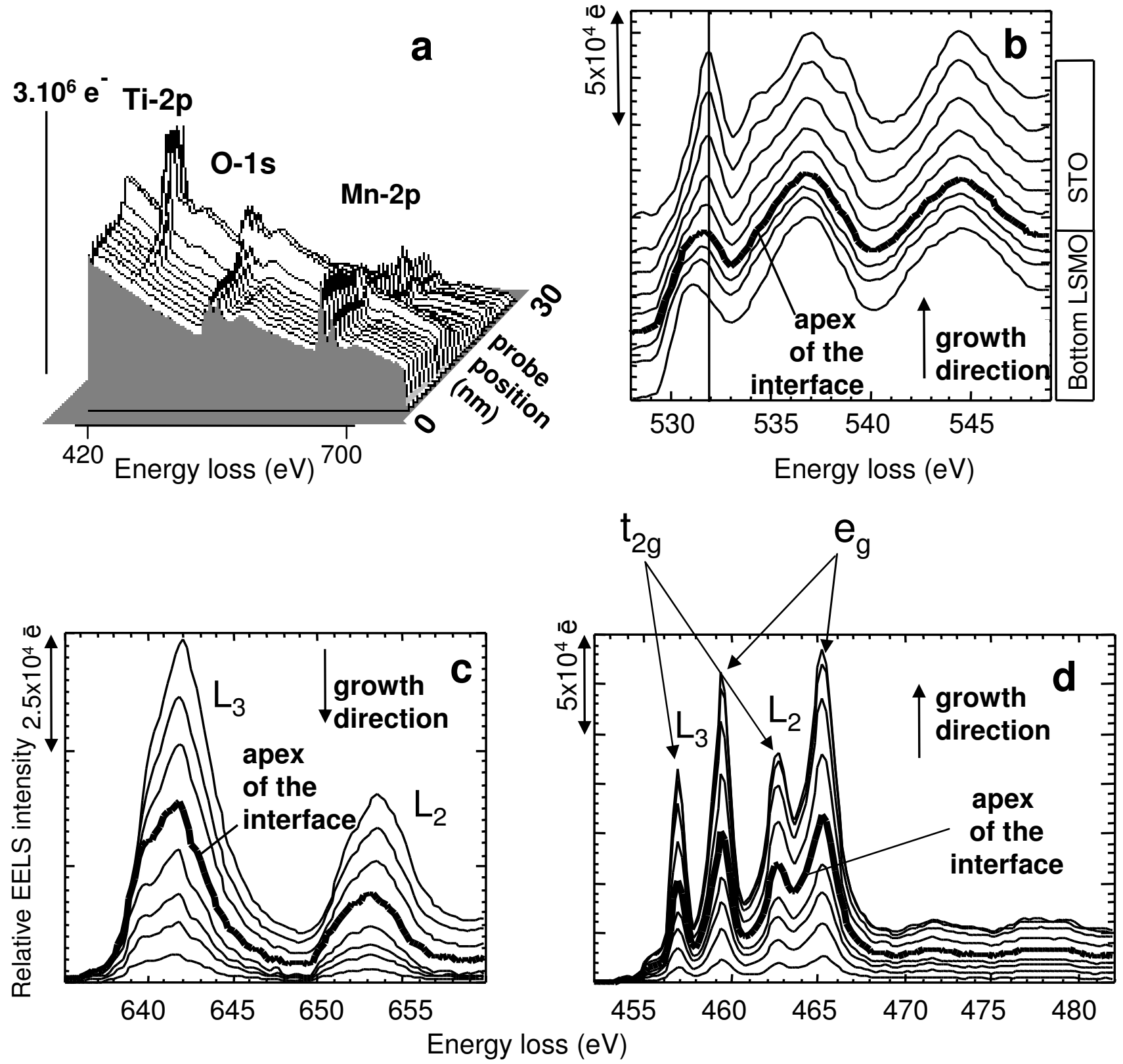

Fig. 6 


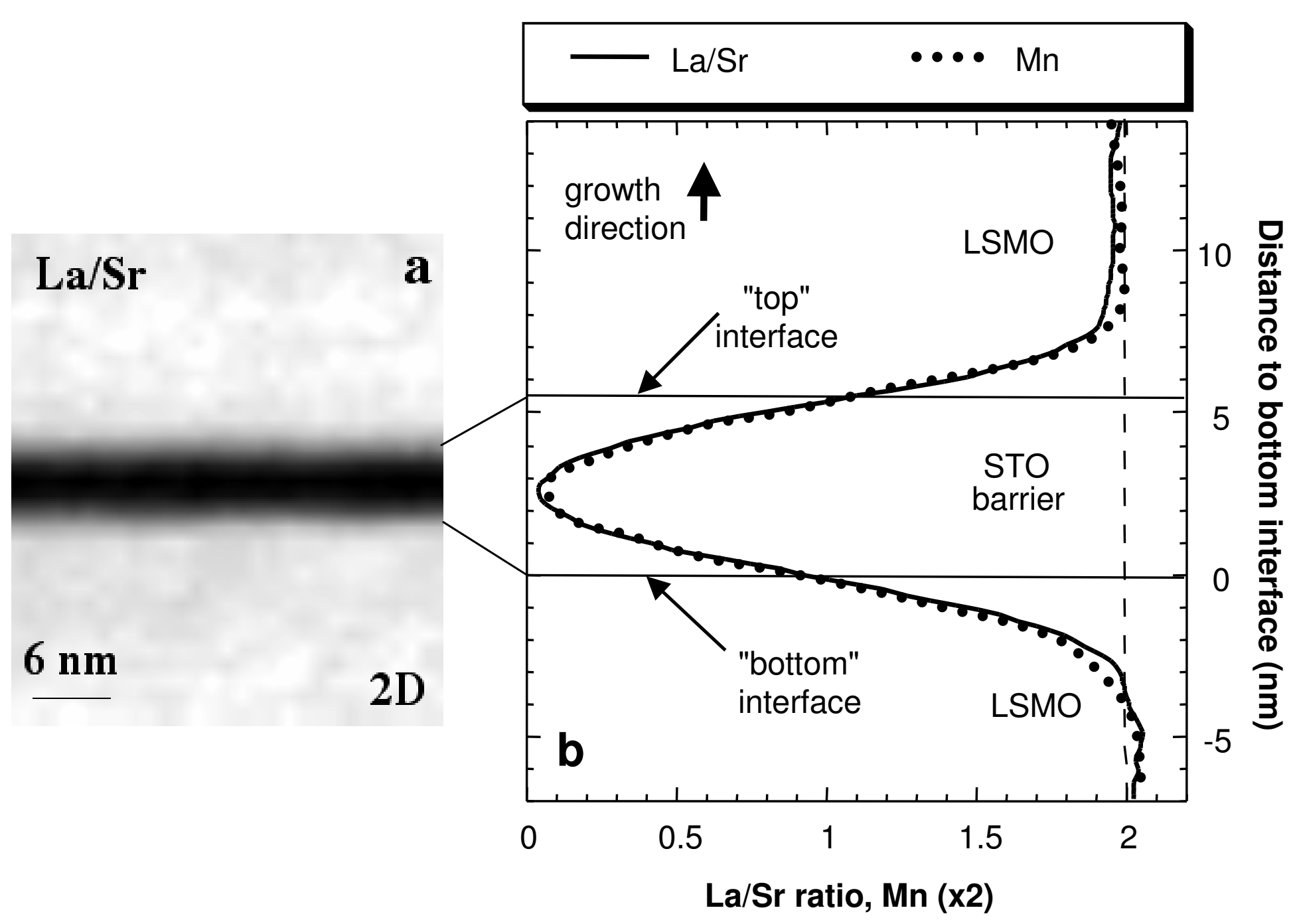

Fig. 7 

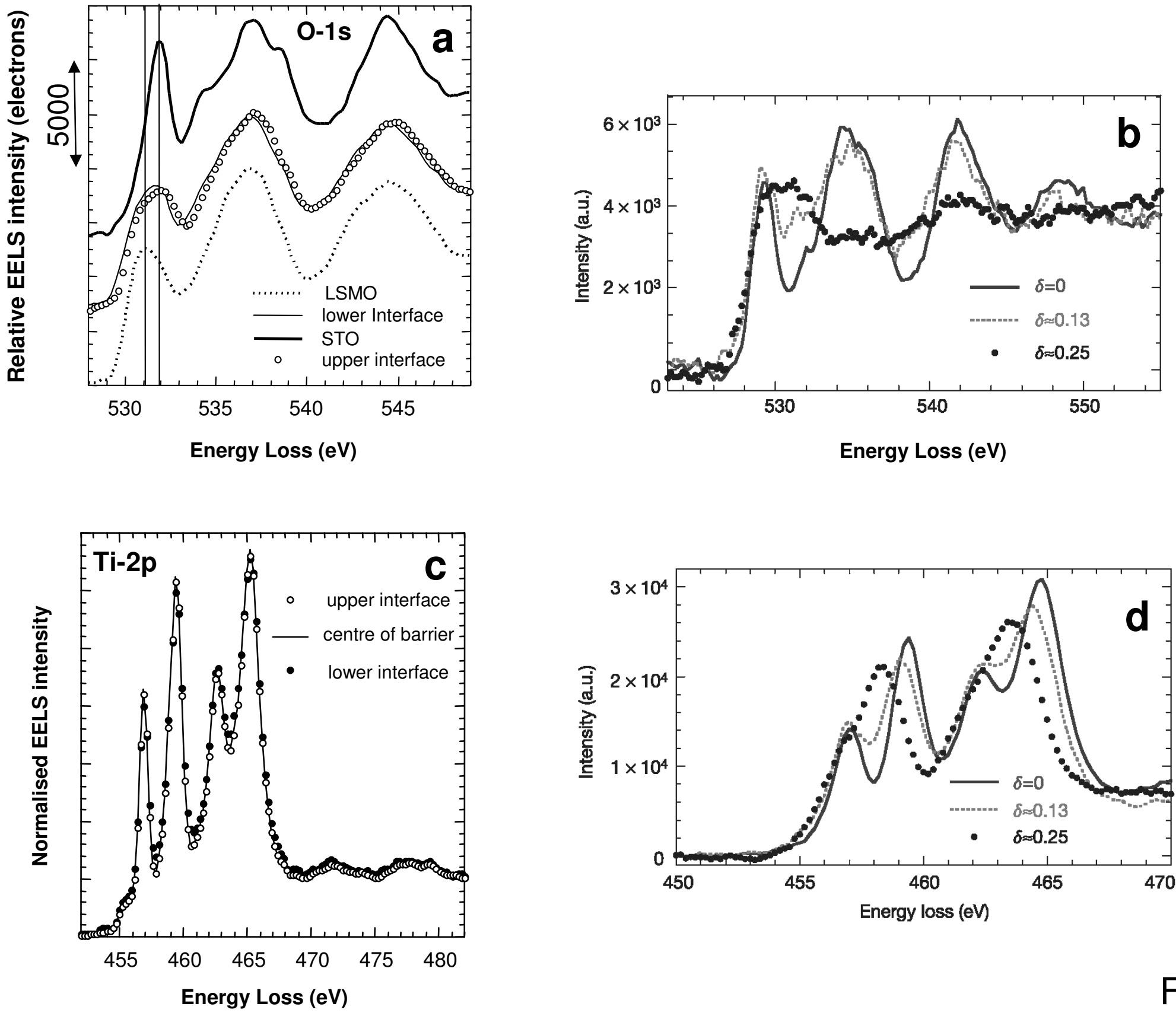

Fig. 8 
Fig. 9

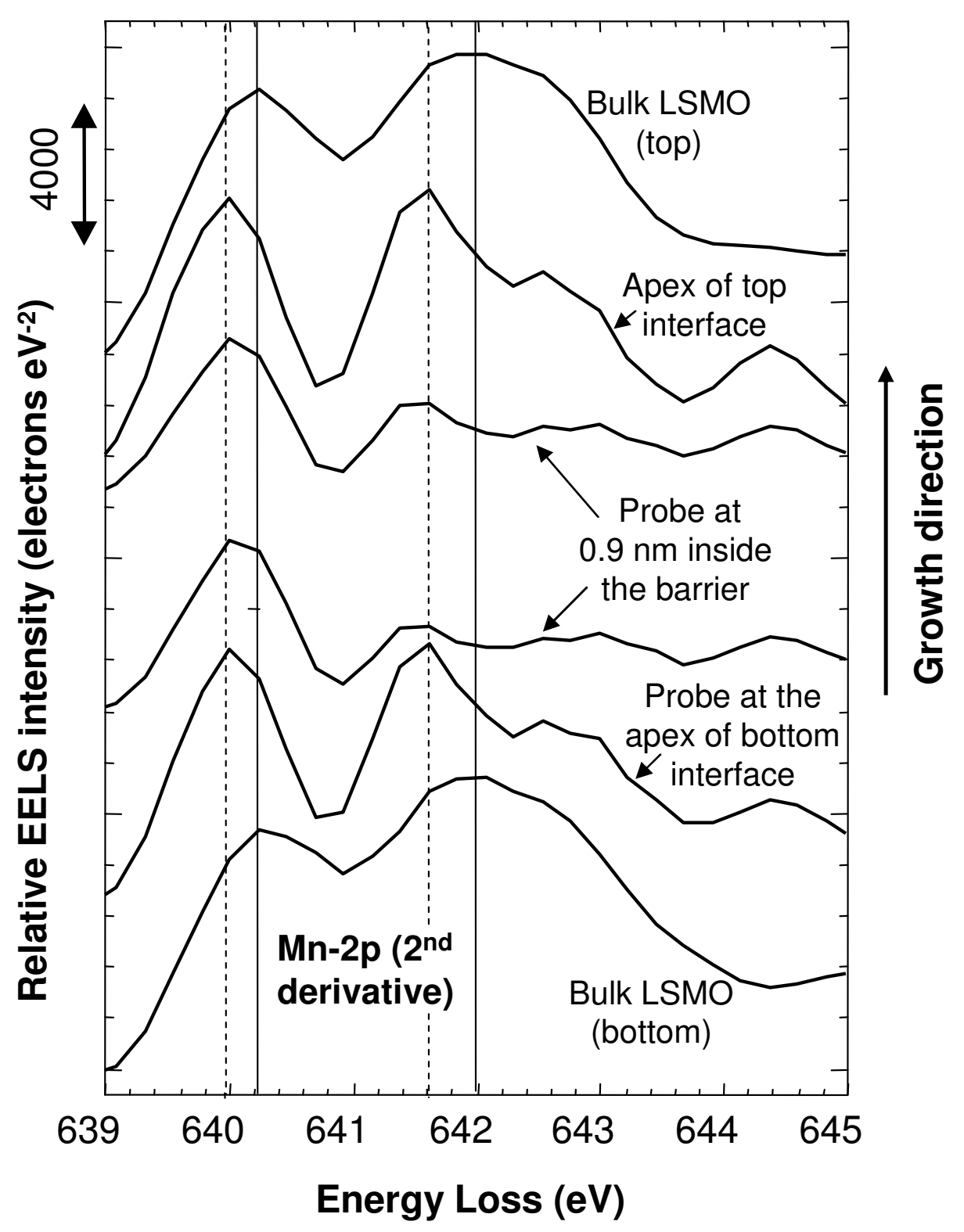

